Corresponding Author:

O.G.Arinola;

Department of Immunology, University of Ibadan, Nigeria +2348023451520

email:

drarinolaog64@yahoo.com

Received 15 June 2020

Accepted 12 August 2020

Published

30 September 2020

Production and Hosting by

Knowledge E

(c) Ganiyu Olatunbosun

Arinola and Fabian Victory

Edem. This article is

distributed under the terms of

the Creative Commons

Attribution License, which

permits unrestricted use and redistribution provided that

the original author and

source are credited.

Editor-in-Chief:

Prof. Mohammad A. M. Ibnouf

\section{Antioxidant Vitamins Are Correlated with Different Aspects of Phagocytic Processes in Healthy Nigerians: Benefits As Supplements During Antimicrobial Treatment}

\section{Ganiyu Olatunbosun Arinola and Fabian Victory Edem}

Department of Immunology, University of Ibadan, Nigeria

\section{Abstract}

Background: Antioxidant vitamins are important for the immune system to function efficiently through several mechanisms. However, according to several previous studies, individual step of leucocyte phagocytosis is not correlated with different antioxidant vitamins.

Methods: This study included 50 healthy Nigerians whosecellular phagocytic mechanism such as percentage leucocyte migration (\%LM) and intracellular killing (\%Nitroblue Tetrazolium Test) were determined by microscopy, neutrophil chemokines [plasma interleukin 8 (IL-8)] was determined using ELISA,and respiratory burst indices [plasma catalase (CAT), superoxide dismutase (SOD), myeloperoxidase (MPO), hydrogen peroxide $\left(\mathrm{H}_{2} \mathrm{O}_{2}\right)$, and nitric oxide (NO)] were determined by spectrophotometry. While the plasma antioxidant vitamins (Vitamins A, C, and E) were determined using HPLC, the phagocytic indices, chemokines, and respiratory burst indices were correlated with plasma antioxidant vitamins using Spearman's Correlation analysis at $\alpha_{0.05}$.

Results: The results show that although among the healthy Nigerian adults, vitamin $C$ was significantly and positively correlated with \%NBT,it was negatively correlated with CAT activity. Vitamin A showed a significantly positive correlation with SOD while Vitamin E showed a significantly negative correlation with MPO.

Conclusions: These findings suggest that antioxidant vitamins affect different stages of phagocytosis. It is advisable to use a combination of antioxidant vitamins as supplements with recommended treatment strategies against intracellular microorganisms or inflammatory diseases.

Keywords: Antioxidants, Intracellular microbial killing, Vitamins

\section{G OPEN ACCESS}

\section{Introduction}

Inadequate intake of antioxidant vitamins suppresses immunity and predisposes to microbial infections that increase morbidity and mortality. It has since been established that the immune system needs vitamins $A, D, C, E, B 6, B 12$, and folate in both innate 
immunity and adaptive immune response to micro-organisms [1]. Phagocytosis constitutes an important innate defense mechanism. During phagocytosis, the appropriate immune cell internalizes micro-organisms through interaction with specific receptors on phagocytic cells. Ultimately, engulfed micro-organisms are digested in phagosomes by hydrolyzing enzymes of the polymorphonuclear leukocytes (PMNL) and macrophages [2]. The SOD enzyme converts superoxide to hydrogen peroxide $\left(\mathrm{H}_{2} \mathrm{O}_{2}\right)$, which is used to produce an oxidant (hypochlorous acid) via myeloperoxidase (MPO). Hypochlorous acid further combines with amines to produce other oxidants chloramines [3]. Phagocytic stages include adherence, movement, engulfment, and reactive oxygen production. A study reported that the entire steps of phagocytosis were increased after the addition of vitamin $\mathrm{E}$ and ascorbic acid in the medium containing peritoneal macrophages [4]. Both phagocytosis and superoxide anion production were enhanced in Kupffer cells treated in vivo with all-trans-retinol [5].

Moreover, while vitamin E deficiency decreased the activity of NK cells, vitamins $\mathrm{C}$ and E reduced the superoxide production by neutrophils [6]. In another study, supplementation with vitamins $C$ and $E$ in healthy humans also demonstrated to suppress neutrophil production of free oxygen radicals [7]. In addition, supplementation of vitamin C also improved antimicrobial and natural killer cell activities, lymphocyte proliferation, chemotaxis, and delayed-type hypersensitivity [6].Alvarado et al.[8]reported that patients with recurrent infections had impaired leukocyte chemotaxis, which was restored with vitamin C. A report provided evidence that a single dose of vitamin C improved primary abnormalities in neutrophil motility and antimicrobial activity in humans [9].

Many aspects of innate immunity are modulated by vitamin A and its metabolites. For instance, vitamin A controls neutrophil maturation, and during vitamin A deficiency, neutrophil numbers were increased but with impaired phagocytic function [10]. Vitamin A also supports phagocytic activity, oxidative burst of macrophages, and bacterial killing [11]. Additionally, itcontrols dendritic cell and CD4 ${ }^{+} \mathrm{T}$ lymphocyte maturation,while vitamin A deficiency diminishes natural killer cell activity and alters the balance between T helper 1 and T helper 2 lymphocytes [12].

Wu et al. [13] demonstrated apositive association between plasma vitamin $E$ and cell-mediated immune responses, and a negative association between plasma vitamin $E$ and the risk of infections in healthy adults. Supplementing adults with vitamin $E$ improved chemotaxis and phagocytosis of neutrophils, natural killer cell activity, and mitogen-induced lymphocyte proliferation [14].

As elucidated earlier, several investigators have suggested that antioxidant vitamins are immunologically beneficial and protective against microbial infections. However, to 
provide additional mechanisms for these earlier observations, the present study determined and correlated separate steps of phagocytosis such as adherence, chemotaxis, digestion, and intracellular killing with antioxidants vitamins.

\section{Methods}

\subsection{Subject population}

This study comprised of 50 healthy subjects who are staff and students in the University College Hospital, Ibadan, Nigeria. While the study included subjects with no communicable and non-communicable disease, those who drink alcoholic beverages, smoke cigarette,and were on compulsory medications or supplements were excluded from the study. All participants gave their consent prior to the studyand the research was conducted in compliance with the Declaration of Helsinki.

\subsection{Plasma isolation}

Blood was collected in a covered test-tube with lithium heparin anticoagulant and carefully mixed. Plasma was removed after centrifuging at $1500 \mathrm{~g}$ for $10 \mathrm{~min}$ in a centrifuge; the liquid component (plasma) was transferred into a clean polypropylene tube using a Pasteur pipette in a freezer $\left(-20^{\circ} \mathrm{C}\right)$.

\subsection{Percentage leucocyte migration}

Percentage leucocyte migration (\%LM) was determined as previously described out in our laboratory by one of the authors [15]. Dextran (6\%) was properly but gently mixed with the whole blood (1:1) and incubated for $45 \mathrm{~min}$ at $37^{\circ} \mathrm{C}$. Supernatant containing the leucocytes was obtained and washed thrice in Kreb-Ringers solution. Washed leucocytes were then filled into capillary tubes and anchored into a migration chamber filled with either Kreb-Ringers solution or antigen (BCG) and Kreb-Ringers solution (1:50) and incubated for $18 \mathrm{hr}$ at $37^{\circ} \mathrm{C}$. The area of $L M$ in the chamber containing antigen was compared with the area of migration in the chamber without antigen. The \%LM was calculated as follows:

$\% \mathrm{LM}=$ (area of migration in antigen solution/area of migration in medium alone) $\times 100$. 


\subsection{Percentage nitroblue tetrazolium dye reduction}

Percentage nitroblue tetrazolium (\%NBT) dye reduction was based on a previously described methodcarried out in our laboratory [15]. For stimulated NBT procedure, NBT solution ( $50 \mu \mathrm{L}$ of $0.2 \% \mathrm{NBT}$ ), heparinized blood ( $25 \mu \mathrm{L}$ ), and $25 \mu \mathrm{L}$ of stimulant solution (nonviable bacterial extract) were mixed gently and incubated at $37^{\circ} \mathrm{C}$ for $10 \mathrm{~min}$. A thick smear of the mixture was prepared which was allowed to air-dry, treated with Wright stain for $15 \mathrm{sec}$, and flooded with distilled water for $30 \mathrm{sec}$ before air-dried. Two hundred leukocytes were counted under oil immersion objective and leukocytes showing dark formazan deposit were recorded as positive. The percentage of bacterially stimulated NBT was calculated as:

$\%$ NBT $=$ [leucocyte with dark formazan deposit (positive)/total leukocytes counted] $\mathrm{x}$ 100.

\subsection{Superoxide dismutase activity determination}

The Superoxide dismutase (SOD) activity was determined as previously carried out in our laboratory by us [44]. This method is based on the principle that SOD inhibits autoxidation of epinephrine at $\mathrm{pH} 10.2$.

\subsection{Catalase activity determination}

Catalase (CAT) activity was determined as previously carried outin our laboratory [44] based on the principle that dichromate in acetic acid is reduced to chromic acetate when heated in the presence of $\mathrm{H}_{2} \mathrm{O}_{2}$, with the formation of perchromic acid as an unstable intermediate. The chromic acetate then produced is measured at $570 \mathrm{~nm}$.

\subsection{Myeloperoxidase activity determination}

Myeloperoxidase (MPO) activity was determined as previously described in our laboratory [44]. The rate of decomposition of $\mathrm{H}_{2} \mathrm{O}_{2}$ by peroxidase, with guaiacol as hydrogen donor, produced tetraguaiacol which was measured at $436 \mathrm{~nm}$. 


\subsection{Hydrogen peroxide determination}

Hydrogen peroxide $\left(\mathrm{H}_{2} \mathrm{O}_{2}\right)$ concentration was determined as previously carried outin our laboratory [44] based on peroxide-mediated oxidation of $\mathrm{Fe}^{2+}$, followed by the reaction of $\mathrm{Fe}^{3+}$ with xylenol orange to form $\mathrm{Fe}^{3+}$-xylenol orange complex with an absorbance maximum of $560 \mathrm{~nm}$. Plasma $\mathrm{H}_{2} \mathrm{O}_{2}$ was determined by comparing absorbance with standard solutions of $\mathrm{H}_{2} \mathrm{O}_{2}$

\subsection{Nitric oxide determination}

Plasma nitric oxide (NO) concentration was determined using Griess reagent [Sulfanilamide and N-1-napthyethylene-diamine dihydrochloride (NED)] as previously described by us [44]. The assay is based on a reaction that utilizes sulfanilamide and NED under acidic (phosphoric acid) conditions. Nitrite forms colored chromophore with reagent, with an absorbance maximum at 540nm.

\subsection{Estimation of chemokine IL-8}

The serum level of IL-8 was determined through Enzyme-linked Immunosorbent Assay (ELISA) using different kits (Abcam, MA, USA, AssayPro, MO, USA and Calbiotech, USA). The ELISA was performed as previously carried out in our laboratory [16].

All the reagents, sample, and standards were allowed to attain working room temperature prior tocommencement of the assay. Stock standard solution was diluted to varying concentration used for the standard curve. Standards, samples, reagents, and immunoplate wereallowed to attain room temperature before being used.After the procedure as described by the manufacturer and as previously carried out, the absorbance of each well was read at $450 \mathrm{~nm}$ with the aid of a microplate reader [SpectraMax 384 Plus (Molecular Devices, USA)]. Using a four-parameter logistic curvefit, the unknown sample concentration was extrapolated from the standard curve.

\subsection{Estimation of serum concentrations of vitamins $A, C$ and $E$ using high-performance liquid chromatography}

Serum vitamins $A$ and $C$ concentrations were determined using High-performance Liquid Chromatography (HPLC) [18]. HPLC is a chromatographic technique used in separating, identifying, and quantifying components in a mixture. It involves passing 
liquid sample oversolid adsorbent material that is packed into a column using a flow of liquid solvent. Analytes in samples interact in a slightly different manner with the adsorbent material, and therebyslowsdown the flow of the analytes differently. A volume of $250 \mu \mathrm{l}$ of standard, controls, and sample were added to $50 \mu l$ internal standard and $250 \mu$ l of precipitating reagent in a $1.5 \mathrm{ml}$ precipitation tube. The mixture was briefly mixed using vortex mixer and leftfor 30 min between $2^{\circ} \mathrm{C}$ and $8^{\circ} \mathrm{C}$, and then centrifuged at $10,000 \mathrm{~g}$ for $2 \mathrm{~min}$ (for vitamin A) or $10 \mathrm{~min}$ (for vitamin C). Supernatant $(100 \mu \mathrm{l})$ was picked and injected into the HPLC-system and the chromatograms weredetected through the UV detector.

\subsection{Statistical analysis}

Spearman's Rank Correlation was used to establish the correlation between \%NBT, \%LM, IL 8, SOD, MPO, CAT, NO, and $\mathrm{H}_{2} \mathrm{O}_{2}$ with antioxidant vitamin levels (vitamins $\mathrm{A}, \mathrm{C}$, and E). Values were considered significant at $p<0.05$.

\section{Results}

Table 1 shows that although vitamin $\mathrm{C}$ was significantly and positively correlated with \%NBT, it was negatively correlated with CATactivity. In addition, while vitamin A showed a significantly positive correlation with SOD, vitamin E showed a significantly negative correlation with MPO.

\section{Discussion}

There are several reports on the role of vitamins in hostingimmunity and susceptibility to infection [6-14]. However, the mechanism is not yet exhausted because it is difficult or rather impossible in humans to study one immune response, single immune cell, or a particular vitamin in isolation due to the synergism of all components of humans. More so, it is difficult to specifically point out if the infection started before vitamin deficiencies orvitamin deficienciescame before the infection. Global threats of infectious diseases [18] coupled with the ongoing global COVID-19 pandemic calls for use of immune boosters, antioxidant, and anti-inflammatory supplements with recommended management regimens.

In children, a close correlation between the deficiency of vitamin A and infectious diseases of respiratory and digestive systems was reported [17, 19]. To some extent, 
TABLE 1: Spearman's Correlation Analysis of \%NBT, \%LM, IL-8, SOD, MPO, CAT, NO, and $\mathrm{H}_{2} \mathrm{O}_{2}$ with antioxidant vitamin levels (vitamins $A, C$, and $E$ )

\begin{tabular}{|c|c|c|c|c|}
\hline & & Vit A & Vit C & Vit E \\
\hline \%NBT & $\begin{array}{l}r \\
p\end{array}$ & $\begin{array}{l}-0.081 \\
0.202\end{array}$ & $\begin{array}{l}0.278 \\
0.000^{*}\end{array}$ & $\begin{array}{l}0.060 \\
0.349\end{array}$ \\
\hline \%LM & $\begin{array}{l}r \\
p\end{array}$ & $\begin{array}{l}-0.066 \\
0.290\end{array}$ & $\begin{array}{l}0.075 \\
0.232\end{array}$ & $\begin{array}{l}0.111 \\
0.076\end{array}$ \\
\hline IL-8 & $\begin{array}{l}r \\
p\end{array}$ & $\begin{array}{l}-0.117 \\
0.062\end{array}$ & $\begin{array}{l}-0.004 \\
0.955\end{array}$ & $\begin{array}{l}-0.061 \\
0.332\end{array}$ \\
\hline SOD & $\begin{array}{l}r \\
p\end{array}$ & $\begin{array}{l}0.136 \\
0.028^{*}\end{array}$ & $\begin{array}{l}0.068 \\
0.272\end{array}$ & $\begin{array}{l}0.104 \\
0.095\end{array}$ \\
\hline MPO & $\begin{array}{l}r \\
p\end{array}$ & $\begin{array}{l}-0.080 \\
0.196\end{array}$ & $\begin{array}{l}-0.044 \\
0.482\end{array}$ & $\begin{array}{l}-0.194 \\
0.002^{*}\end{array}$ \\
\hline CAT & $\begin{array}{l}r \\
p\end{array}$ & $\begin{array}{l}-0.011 \\
0.862\end{array}$ & $\begin{array}{l}-0.168 \\
0.007^{*}\end{array}$ & $\begin{array}{l}-0.006 \\
0.929\end{array}$ \\
\hline $\mathrm{H}_{2} \mathrm{O}_{2}$ & $\begin{array}{l}r \\
p\end{array}$ & $\begin{array}{l}0.068 \\
0.276\end{array}$ & $\begin{array}{l}0.068 \\
0.272\end{array}$ & $\begin{array}{l}-0.007 \\
0.908\end{array}$ \\
\hline NO & $\begin{array}{l}r \\
p\end{array}$ & $\begin{array}{l}0.150 \\
0.016^{*}\end{array}$ & $\begin{array}{l}0.022 \\
0.724\end{array}$ & $\begin{array}{l}0.108 \\
0.083\end{array}$ \\
\hline
\end{tabular}

*Significantly correlated

vitamin A demonstrated a therapeutic effect in diseases transmitted through the respiratory system such as pneumonia and measles in children [20]. In rodents, deficiency of vitamin A reduced the adhesion and phagocytosis of Pseudomonas aeruginosa and the production of reactive oxidative molecules [21]. Epidemiological studies showed that the healthy population had a significantly higher serum level of vitamin A than pulmonary tuberculosis patients $[22,23]$. The suggested immunological effect was based on the fact that vitamin $A$ is a micronutrient that protects epithelium integrity [18-20] and plays a crucial role in mucus production, epithelial-formation, -keratinization, -stratification, -differentiation, and functional maturation [24].

Antioxidant enzymes such as SOD and CAT act as a defense against oxidative stress [25]. SOD catalyzes the conversion of a highly reactive superoxide anion $\left(\mathrm{O}_{2}^{--}\right)$ into a less reactive species $\mathrm{H}_{2} \mathrm{O}_{2}$ and molecular oxygen $\left(\mathrm{O}_{2}\right) . \mathrm{H}_{2} \mathrm{O}_{2}$ formed by SOD activity is decomposed to water and molecular oxygen $\left(\mathrm{O}_{2}\right)$, a reaction catalyzed by the enzyme CAT [26]. In the present study, vitamin A had a positive correlation with SOD, thus increasingthe levels of vitamin A and thereby increasing the SOD activities and production of antimicrobial $\mathrm{H}_{2} \mathrm{O}_{2}$. This might be one of the bases for the protective uses of vitamin A.

Vitamin C deficiency increases susceptibility to a variety of infections [27] and humansdeficient in vitamin C are susceptible to pneumonia [28]. Vitamin C supplementation decreased the duration and severity of common cold [29]. Pulmonary tuberculosis patients have decreased plasma vitamin C concentrations compared 
withuninfectedsubjects [30]. Based on chest X-ray results, acute lung infections have shown rapid clearance following an administration of intravenous vitamin $C[31,32]$. In addition, pre -clinical studies of animals with sepsis-induced lung injury have indicated that vitamin $\mathrm{C}$ administration increased alveolar fluid clearance, enhanced bronchoalveolar epithelial barrier function, attenuated sequestration of neutrophils [33].

Immunomodulatory function of vitamin $\mathrm{C}$ was based on its requirement for the biosynthesis of collagen, maintenance of epithelial integrity, leucocyte chemotaxisin response to inflammatory mediators, phagocytosis and killing of microbes, apoptosis, and clearance by macrophages [34]. Vitamin $\mathrm{C}$ has effective antioxidant capacitybecause of its ability to donate electrons, thus protecting important biomolecules (such as proteins, lipids, carbohydrates, and nucleic acids) from damage by oxidants generated during normal cellular activities [35]. In addition to these previously suggestedmechanisms, the present study added that positive correlation of NBT (ability to generate reactive oxygen species) or negative correlation of CAT (another antioxidant enzyme) with vitamin C levels might also contribute to the usefulness of vitamin $C$ in immune function. Our findings are in support of earlier reports that state leukocytes actively accumulate vitamin $C$ [36], which increases following oxidative burst [37] and that vitamin $C$ is a water-soluble antioxidant that scavenges numerous oxidants and can also regenerate important cellular and membrane antioxidants such as glutathione and vitamin E [38]. Another study by Bozonet and Carr [39] also demonstrated that supplementation with vitamins $C$ and $E$ in healthy humans suppressed neutrophil production of free oxygen radicals.Moreover, the supplementation of elderly women with a combination of $1 \mathrm{~g} / \mathrm{day}$ vitamin C with vitamin E enhanced neutrophil functions, including chemotaxis [40]. This observation therefore supports the use of combination of antioxidant vitamins

Further, vitamin E was shown toreduce susceptibility to infections by enhancingproliferation of lymphocytes, production of T helper 1-type cytokines, activities of natural killer cells, and phagocytosis of macrophages/neutrophils [41]. It was shown thatthe positive association existed between plasma vitamin $\mathrm{E}$ and cell-mediated immune responses, and a negative association has been demonstrated between plasma vitamin $E$ and the risk of infections in healthy adults over 60 years of age $[42,43]$.

During the respiratory burst, leucocytes consume a large amount of $\mathrm{O}_{2}$ to generate $\mathrm{H}_{2} \mathrm{O}_{2}$ through dismutation reaction catalyzed by SOD [25]. This $\mathrm{H}_{2} \mathrm{O}_{2}$ combines with halide ions $\left(\mathrm{I}^{-}, \mathrm{Cl}^{-}, \mathrm{Br}^{-}\right)$to produce hypohalous acid $(\mathrm{HOCl} / \mathrm{HOBr})$ in the presence of MPO. $\mathrm{HOCl} / \mathrm{HOBr}$ may react with $\mathrm{O}_{2} \cdot{ }^{--}$or $\mathrm{Fe}^{2+}$ to form another strong oxidant, probably the hydroxyl radical $(\cdot \mathrm{OH})$. If not controlled and due to high concentrations, the reactive oxygen species $\left(\mathrm{O}_{2} \cdot{ }^{-}, \cdot \mathrm{OH}\right), \mathrm{HOCl} / \mathrm{HOBr}$, and $\mathrm{H}_{2} \mathrm{O}_{2}$ may leak into surrounding 
cells resulting in increased quantities of free radicals [26]. This study shows a negative correlation between vitamin E and MPO supporting that vitamin E is an antioxidant vitamin. It is thus likely that vitamin E is consumed as MPO activity increases so as to reduce the excessive production of $\mathrm{HOCl} / \mathrm{HOBr}$ and subsequent stronger oxidants like $\mathrm{O}_{2} \cdot{ }^{-}$and $\cdot \mathrm{OH}$.

Therefore, according to this study, antioxidant vitamins A, C, and E support different stages of phagocytosis and the three antioxidant vitamins are thusrecommended to be taken together as supplements in the treatment of microbial and inflammatory diseases.

\section{References}

[1] EFSA Panel on Dietetic Products, Nutrition and Allergies (NDA). (2016). Guidance on the scientific requirements for health claims related to the immune system, the gastrointestinal tract and defense against pathogenic microorganisms. EFSA Journal, vol. 14, p. 4369.

[2] Aderem, A. andUnderhill, D. M. (1999).Mechanisms of phagocytosis in macrophages. Annual Review of Immunology, vol. 17, pp. 593-623.

[3] Winterbourn, C.C., Kettle, A.J., and Hampton, M.B. (2016). Reactive oxygen species and neutrophil function. Annual Review of Immunology, vol. 85, pp. 765-792.

[4] Del Rio, M., Ruedas, G., Medina, S., et al. (1998).Improvement by several antioxidants of macrophage function in vitro. Life Sciences, vol. 63, pp. 871-881.

[5] Hoglen, N. C., Abril, E. A., Sauer, J. M., et al. (1997). Modulation of Kupffer cell and peripheral blood monocyte activity by in vivo treatment of rats with all-transretinol.Liver, vol. 17, pp. 157-165.

[6] de la Fuente, M., Ferrandez, M. D., Burgos, M. S., et al. (1998). Immune function in aged women is improved by ingestion of vitamins C and E. Canadian Journal of Physiology and Pharmacology, vol. 76, pp. 373-380.

[7] Sureda, A., Ferrer, M. D., Mestre, A., et al.(2013). Prevention of neutrophil protein oxidation with vitamins $C$ and $E$ diet supplementation without affecting the adaptive response to exercise. International Journal of Sport Nutrition and Exercise Metabolism, vol. 23, no. 1, pp. 31-39.

[8] Alvarado, C., Alvarez, P., Puerto, M., et al.(2006). Dietary supplementation with antioxidants improves functions and decreases oxidative stress of leukocytes from prematurely aging mice. Nutrition, vol. 22, no. 7-8, pp. 767-777.

[9] Wintergerst, E. S., Maggini, S., and Hornig, D. H.(2006). Immune-enhancing role of vitaminC and zinc and effect on clinical conditions. Annals of Nutrition and 
Metabolism, vol. 50, no. 2, pp. 85-94.

[10] Shrestha, S., Kim, S.Y., Yun, Y.J., et al.(2017). Retinoic acid induces hypersegmentation and enhances cytotoxicity of neutrophils against cancer cells. Immunology Letters, vol. 182, pp. 24-29.

[11] Vellozo, N.S., Pereiramarques, S.T., Cabralpiccin, M.P., et al. (2017). All-trans retinoic acid promotes an m1- to m2-phenotype shift and inhibits macrophage-mediated immunity to leishmania major. Frontiers in Immunology, vol. 8, p. 1560.

[12] Kiss, I., Rühl, R., Szegezdi, E., et al. (2008). Retinoid receptor-activating ligands are produced within the mouse thymus during postnatal development. European Journal of Immunology, vol. 38, pp. 147-155.

[13] Wu, D., Han, S. N., Meydani, M., et al.(2006). Effect of concomitant consumption of fish oil and vitaminE on T cell-mediated function in the elderly: a randomized double-blind trial. Journal of the American College of Nutrition, vol. 25, no. 4, pp. 300-306.

[14] De la Fuente, M.,Hernanz, A., Guayerbas, N., et al.(2008). Vitamin E ingestion improves several immune functions in elderly men and women. Free Radical Research, vol. 42, pp. 272-280.

[15] Arinola, O. G. and Salimonu, L. S. (1999). Leukocytes migration inhibitory factor assay in Nigerians with urinary schistosomiasis. West African Journal of Medicine, vol. 18, p. $101 e 5$

[16] Arinola, O.G., Yaqub, S., and Rahamon, S.K. (2012). Reduced serum IgE level in Nigerian children with Nigerian children with helminthiasis compared with protozoan infection: Implication on hygiene hypothesis. Annal of Biological Research, vol.3, no. 12, pp. 5754-5757.

[17] Edem, V. F., Ige, O. M., and Arinola, O. G. (2016). Plasma vitamins and essential trace elements in multi-drug resistant pulmonary tuberculosis patients before and during chemotherapy. Egyptian Journal of Chest Diseases and Tuberculosis, vol. 65, no. 2, pp. 441-445.

[18] Rytter, M.J., Kolte, L., Briend, A., et al. (2014). The immune system in children with malnutrition-A systematic review. PLoS ONE, vol.9, e105017.

[19] Tan, P.H., Sagoo, P., Chan, C., et al. (2005). Inhibition of NF-kappa B and oxidative pathways in human dendritic cells by antioxidative vitamins generates regulatory $T$ cells. Journal of Immunology, vol. 174, pp. 7633-7644.

[20] Lammermann, T. (2016). In the eye of the neutrophil swarm-navigation signals that bring neutrophils together in inflamed and infected tissues. Journal of Leukocyte Biology, vol. 100, pp. 55-63. 
[21] Twining, S. S., Schulte, D. P., Wilson, P. M., et al. (1997). Vitamin A deficiency alters rat neutrophil function. Journal of Nutrition, vol. 127, pp. 558-565.

[22] Blass, S.C., Goost, H., Tolba, R.H., et al. (2012). Time to wound closure in trauma patients with disorders in wound healing is shortened by supplements containing antioxidant micronutrients and glutamine: A PRCT. Clinical Nutrition, vol. 31, pp. 469475.

[23] Martin, P. and Leibovich, S.J. (2005). Inflammatory cells during wound repair: The good, the bad and the ugly. Trends in Cell Biology, vol. 15, pp. 599-607.

[24] Amitromach, E., Uni, Z., Cheled, S., et al. (2009). Bacterial population and innate immunity-related genes in rat gastrointestinal tract are altered by vitamin A-deficient diet. Journal of Nutritional Biochemistry, vol. 20, pp. 70-77.

[25] Prasanthi, K. and Rajini, P. S. (2005). Morphological and biochemical perturbations in rat erythrocytes following in vitro exposure to Fenvalerate and its metabolite. Toxicology in Vitro, vol.19, pp. 449-456.

[26] Ruas, C. B., Carvalho Cdos, S., de Araújo, H. S., et al. (2008). Oxidative stress biomarkers of exposure in the blood of cichlid species from a metal-contaminated river. Ecotoxicology and Environmental Safety, vol.71, pp. 86-93.

[27] Hemilä, H.(2017).Vitamin C and infections. Nutrients, vol. 9, p. 339.doi:10.3390/nu9040339.

[28] Hemilä, H. and Louhiala, P. (2013). Vitamin C for preventing and treating pneumonia. Cochrane Database of Systematic Reviews. Retrieved from: https: //www.cochranelibrary.com/cdsr/doi/10.1002/14651858.CD005532.pub3/full

[29] Hemilä, H. and Chalker, E. (2013).Vitamin C for preventing and treating the common cold. Cochrane Database of Systematic Reviews. Retrieved from: https://www. cochranelibrary.com/cdsr/doi/10.1002/14651858.CD000980.pub4/full

[30] Bakaev, V.V. and Duntau, A.P. (2004). Ascorbic acid in blood serum of patients with pulmonary tuberculosis and pneumonia. International Journal of Tuberculosis and Lung Disease, vol. 8, pp. 263-266.

[31] Bharara, A., Grossman, C., Grinnan, D., et al. (2016). Intravenous vitamin C administered as adjunctive therapy for recurrent acute respiratory distress syndrome. Case Reports in Critical Care, 8560871.

[32] Fowler, A.A., Kim, C., Lepler, L., et al. (2017). Intravenous vitamin C as adjunctive therapy for enterovirus/rhinovirus induced acute respiratory distress syndrome. World Journal of Critical Care Medicine, vol. 6, pp. 85-90. 
[33] Fisher, B.J., Kraskauskas, D., Martin, E.J., et al. (2012). Mechanisms of attenuation of abdominal sepsis induced acute lung injury by ascorbic acid. American Journal of Physiology-Lung Cellular and Molecular Physiology, vol. 303, pp. L20-L32.

[34] Wilgus, T.A., Roy, S., and McDaniel, J.C. (2013). Neutrophils and wound repair: Positive actions and negative reactions. Advances in Wound Care, vol. 2, pp. 379-388.

[35] Carr, A. and Frei, B. (1999). Does vitamin C act as a pro-oxidant under physiological conditions? FASEB Journal, vol. 13, pp. 1007-1024.

[36] Bergsten, P., Amitai, G., Kehrl, J., et al. (1990). Millimolar concentrations of ascorbic acid in purified human mononuclear leukocytes. Depletion and reaccumulation. Journal of Biological Chemistry, vol. 265, pp. 2584-2587.

[37] Corpe, C.P., Lee, J.H., Kwon, O., et al. (2005). 6-Bromo-6-deoxy-I-ascorbic acid: An ascorbate analog specific for $\mathrm{Na}^{+}$-dependent vitamin $\mathrm{C}$ transporter but not glucose transporter pathways. Journal of Biological Chemistry, vol. 280, pp. 5211-5220.

[38] Buettner, G.R. (1993). The pecking order of free radicals and antioxidants: Lipid peroxidation, alpha-tocopherol, and ascorbate. Archives of Biochemistry and Biophysics, vol. 300, pp. 535-543.

[39] Bozonet, S. M. and Carr, A. C. (2019). The role of physiological vitamin concentrations on key functions of neutrophils isolated from healthy individuals. Nutrients, vol. 11, no. 6 , p. 1363.

[40] Nusgens, B.V., Humbert, P., Rougier, A., et al. (2001). Topically applied vitamin C enhances the mRNA level of collagens I and III, their processing enzymes and tissue inhibitor of matrix metalloproteinase 1 in the human dermis. Journal of Investigative Dermatology,vol. 116, pp. 853-859.

[41] Wu, D. and Meydani, S. N. (2004). Age-associated changes in immune function: impact of vitamin $\mathrm{E}$ intervention and the underlying mechanisms. Endocrine, Metabolic \& Immune Disorders - Drug Targets, vol. 14, pp. 283-289.

[42] Lee, G. andHan, S. (2018). The role of vitamin E in immunity. Nutrients, vol.10, p. 614

[43] Meydani, S. N., Han, S. N., and Wu, D. (2005). Vitamin E and immune response in the aged: Molecular mechanisms and clinical implications. Immunological Reviews, vol. 205, pp. 269-284.

[44] Akinyoola, S. B., Edem, V. F., Arinola, O. G., et al.(2012). Vitamin E or red palm oil increases the level of nitric oxide in Wister rats chronically exposed to dichlorvos. Tropical Journal of Health Sciences, vol. 19, p. 8e11. 